Scientific Paper

\title{
Low cost blood vein detection system based on near-infrared LEDs and image-processing techniques
}

\author{
Mohammed J. ALWAZZAN ${ }^{1, a}$ \\ ${ }^{I}$ Department of Medical Instrumentation Techniques Engineering, AL-Hussain University College, Karbala, Iraq \\ ${ }^{a}$ E-mail address: dr.mohammedjamal@huciraq.edu.iq
}

(received 30 October 2019; revised 21 January 2019; accepted 11 March 2020)

\begin{abstract}
Drawing blood and injecting drugs are common medical procedures, for which accurate identification of veins is needed to avoid causing unnecessary pain. In this paper, we propose a low-cost system for the detection of veins. The system emits near-infrared radiation from four light-emitting diodes (LEDs), with a charge-coupled device (CCD) camera located in the middle of the LEDs. The camera captures an image of the palm of the hand. A series of digital imageprocessing techniques, ranging from image enhancement and increased contrast to isolation using a threshold limit based on statistical properties, are applied to effectively isolate the veins from the rest of the image.
\end{abstract}

Key words: NIR LEDs; CCD camera; palm vein; Wiener filter; CLAHE.

\section{Introduction}

A major problem faced by medical personnel is difficulty in accessing veins for drug delivery or taking blood samples for tests [1]. In many cases, even experienced paramedics or nurses are unable to reach veins in patients whose veins are unclear or invisible, especially in the obese, the elderly, care patients, and children [2]. Poor visibility leads to unnecessary puncturing of veins, causing problems such as bruises, wounds, rashes, blood clots, or permanent damage to the vein $[3,4]$. In these cases, it becomes necessary to have a non-invasive and easy to perform veins detection system [5]. The skin consists of three main layers: the epidermis, dermis, and hypodermis [6]. The epidermis absorbs a portion of the light, and the light travels through the epidermis to the tissue beneath it, after which it scatters [7]. Most scattering occurs within the dermis layer before it spreads to the hypodermis, but part of the light is absorbed [8]. The subcutaneous fat scatters a large portion of light and absorbs a small portion of it. A small portion of the light that reaches the blood is absorbed into the blood vessels by the hemoglobin in the blood, but a large part of it scatters due to the relatively large size of red blood cells [7,9]. Haemoglobin absorbs light in the near-infrared (NIR) wavelength, specifically at wavelengths from 740-760 $\mathrm{nm}$ [10]. Intravenous blood contains deoxyhaemoglobin at a concentration greater than that of haemoglobin: the concentration of haemoglobin in veins is approximately $47 \%$, whereas the concentration of haemoglobin in arteries is 9095\% [11]. The two types of haemoglobin have different light absorption properties, as shown in Figure 1.

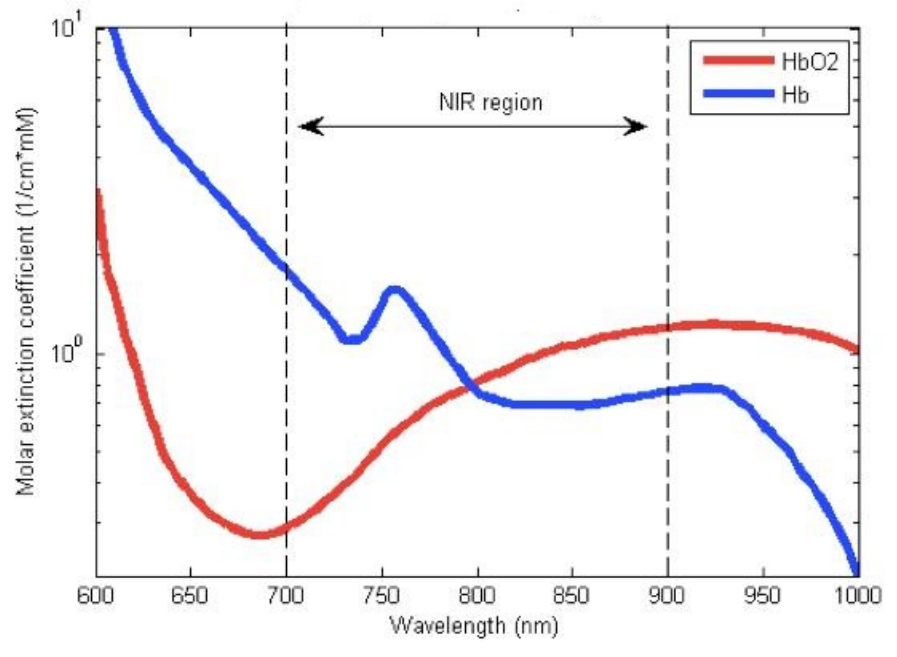

Figure 1. Absorption spectrum of haemoglobin (Hb) [12].

The absorption of light by the veins is higher than the absorption of light by the arteries between the wavelengths of 600 to 800 nanometers, as it decreases rapidly with respect to deoxyhaemoglobin while it rises slightly and then decreases with respect to haemoglobin [13]. Wavelengths from 300 to $400 \mathrm{~nm}$ reach only the epidermis and certain sections of the dermis that do not contain any veins [14]. Infrared radiation, whose wavelength is between 700 and 1000 nanometers, reaches the blood vessels of the base layer. The vein devices work by highlighting the patient's skin using bright lightemitting diodes (LEDs) to display the veins for access [15]. The vein pattern can be detected with help of infrared sensors [16]. 
Two different imaging techniques, based on far-infrared (FIR) and near-infrared radiation [17], can be used. Vein detection systems that use FIR produce low-contrast images, rendering it difficult to distinguish veins from other components of the acquired image [18]. NIR rays penetrate the tissue easily; thus, they give clearer images than FIR [19]. Over the past several decades, many devices and systems have been developed to help nurses and doctors find veins and avoid pointless twitching. Chandra et al. designed a system for detecting veins at the visible spectrum in a wavelength range of 600-696 nm. Although this system was effective in distinguishing the colour of the skin, it was not able to locate the veins accurately, and it lacked the software to locate the veins [20]. Miura et al. used a suggested technique to detect the veins of the fingers using a visible light camera and a series of digital image-processing techniques; the technique proposed by them was economical and inexpensive, but it did not isolate the veins from the other image details [21]. Ahmed et al. used an infrared camera and captured images in real-time and improved them using contrast limited adaptive histogram equalization (CLAHE); the design that was used in this system was somewhat expensive, especially since the thermal camera is expensive. In addition to this, they merely improved the image; they did not isolate the veins from the rest of the details of the captured image [18]. Prijono et al designed a system for detecting veins based on a camera and an infrared filter; they were able to isolate the veins from the rest of the image details using modified local line binary pattern (MLLBP), but the isolated veins were not accurate, and the veins were wide [22]. From previous studies, we note that the vein detection techniques used in them fail due to their high cost, lack of accurate software for detecting veins, and the problem of wide veins; thus, there is no device that solves the above problems together. In this paper, we suggest a low-cost system that relies on infrared LEDs and a series of digital image-processing techniques so that it can detect veins accurately and solve the problem of wide vein isolation.

\section{Materials and Methods}

\section{Materials used}

The prototype developed here uses an optional imaging technique to improve the visibility of the veins in the dermis layer of the skin. The device irradiates the patient's palm, placed on a stationary base at a fixed distance from the chargecoupled device (CCD) camera, with an NIR wavelength of $800 \mathrm{~nm}$ (Figure 2). The camera is able to detect infrared wavelengths that the human eye cannot. A USB cable connected between the device and a computer supplies power and allows the digital image to be transferred to the computer and processed using MATLAB.

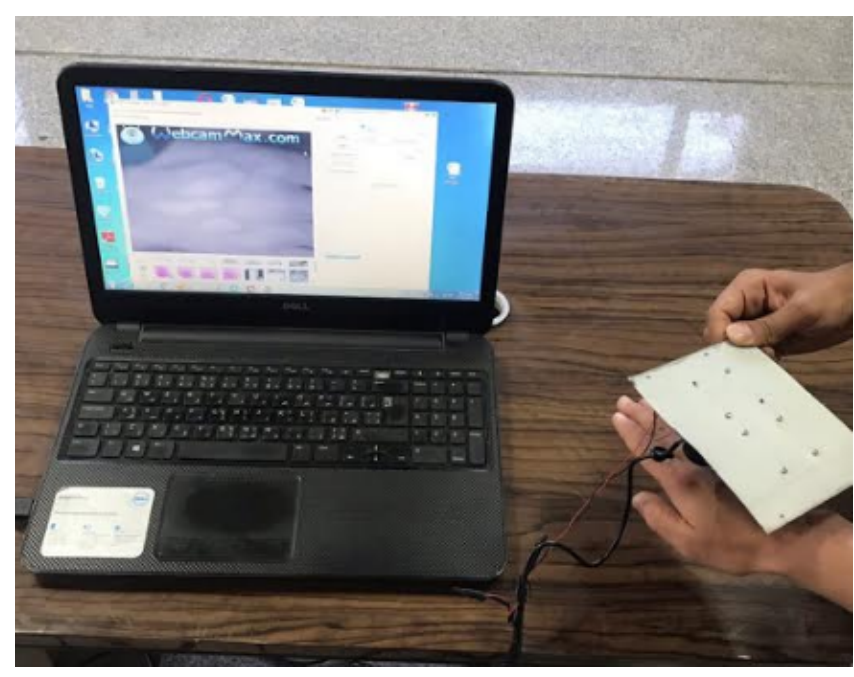

Figure 2. The proposed system.

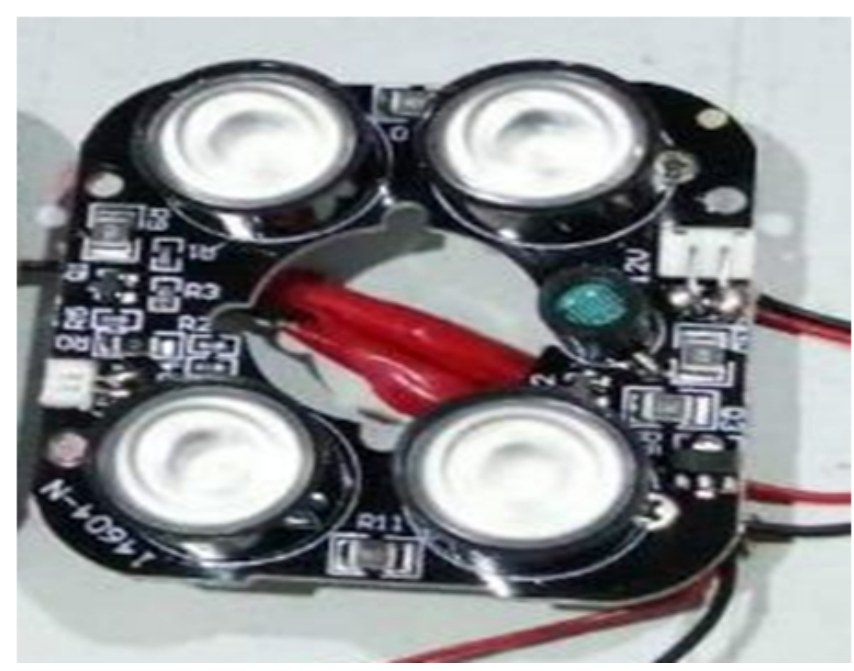

Figure 3. NIR LEDs used for illumination.

Various distances from the camera to the palm $(5 \mathrm{~cm}, 10 \mathrm{~cm}$, $15 \mathrm{~cm}, 20 \mathrm{~cm}, 25 \mathrm{~cm}$, and $30 \mathrm{~cm}$ ) were selected to find the ideal distance for venous quality. The camera captures an image of the reflected radiation from the skin, which passes through a converter to produce a digital image. The camera was chosen for its ideal matrix size of 10-30 micrometers and the ability to obtain a signal even in complete darkness, as the intensity of the signal depends on the time of exposure and the temperature of the camera. The camera is mounted in the middle of a $10 \times 15 \mathrm{~cm}$ panel, and four NIR LEDs with a wavelength of $800 \mathrm{~nm}$ are placed around the camera, as depicted in Figure 3. This arrangement of LEDs around the camera gives an effective distribution of lighting.

The images were processed using MATLAB. The aim of this project was not to create a commercial prototype but rather to find the processing algorithm that demonstrated the best results for vein identification. 


\section{Blood vein detection algorithm}

The software used for developing the vein detection algorithm was MATLAB R2012b. The algorithm can be summarized in six steps (Figure 4):

1. Read the input image acquired by shining near-infrared radiation on the palm of a subject. Figure 5 shows the colour image acquired by the system.

2. Convert the red, green, and blue (RGB) image into a greyscale image by applying specific weights to the red, green, and blue channels. These weights were $0.2989,0.5870$, and 0.1140 , respectively. The conversion equation has the following form [23]:

$$
0.2989 * R+0.5870 * G+0.1140 * B
$$

3. Enhance the greyscale image using a Wiener filter. Image enhancement serves the important purpose of removing noise from the image. Noise may come from the camera sensor or may be introduced during image transfer and storage [24]. The image is further enhanced by reducing or removing blur from the image. The Wiener filter is a low-pass filter that depends on the colour density of the image and uses the mean and standard deviation to determine which pixels will be replaced with the centre of the window within the field of adjacent pixels [25].

4. Apply the CLAHE technique to enhance the contrast of the image.

5. Carry out automatic image segmentation. Image segmentation is meant to separate distinct elements in an image from other elements [26]. After these distinctive elements have been separated, further operations can be performed, such as identifying individual elements or measuring their size. We suggest a threshold based on the statistical properties of the image, in the following form:

$$
T=2 \alpha-\min +(255-\max )
$$

Image segmentation (i.e. generating a binary image) can be performed using the proposed threshold:

$$
g(x, y)=\left\{\begin{array}{l}
255 \text { if } f(x, y)<T \\
0 \quad \text { otherwise }
\end{array}\right.
$$

where $g(x, y)$ is the binary output image, $f(x, y)$ is the input image, $\alpha$ is the standard deviation, $\min$ is the minimum value, and max is the maximum value.

6. Apply a series of morphological processes to eliminate unwanted pixels. The morphological processes provided by the MATLAB environment will include choosing the largest compound to eliminate unwanted pixels that do not represent the vein area by using the connected vehicle algorithm and eliminating the wide vein by using the strel function [25].

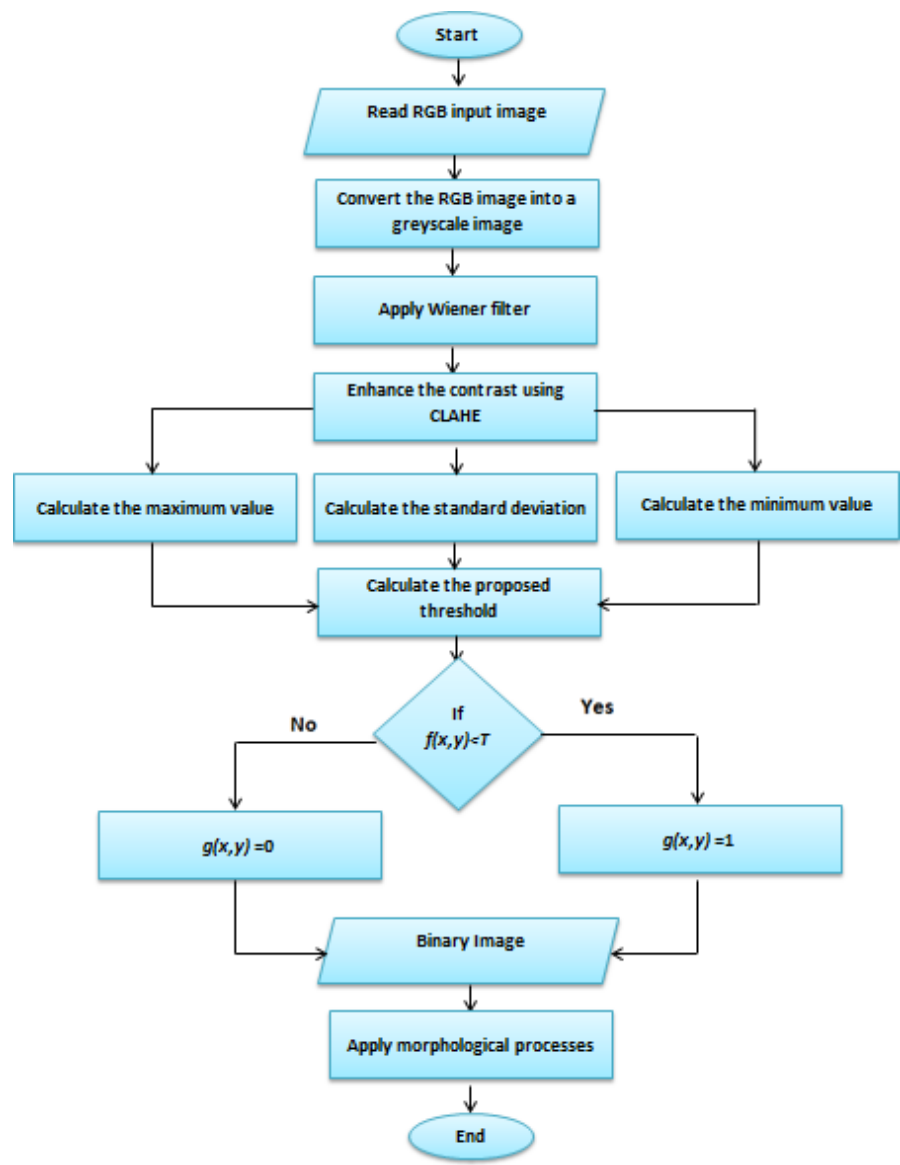

Figure 4. Illustration of the basic steps of the proposed algorithm.

\section{Results and discussion}

The image acquired from the prototype vein detection system is a 24-bit colour image, which is converted to greyscale (8 bit). Colour images have the ability to store more information than greyscale images; thus, the colour image is larger than the greyscale image, leading to a longer processing time [25]. Figure 5 and $\mathbf{6}$ show colour and greyscale images acquired by the system.

All the images obtained by the proposed system were cleaned with water and soap and then sterilized using alcohol before the image was taken by the proposed system; this was to remove the oily layer from the skin, as the oily layer on the skin causes an unwanted spectral reflection that negatively affects the quality of the image captured with the proposed system [27].

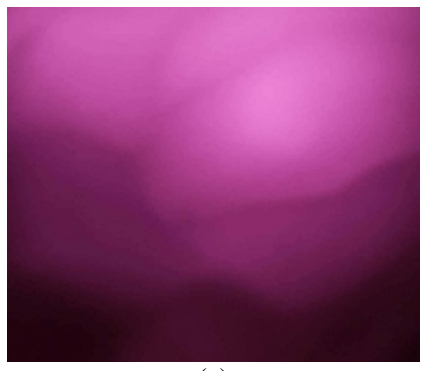

(a)

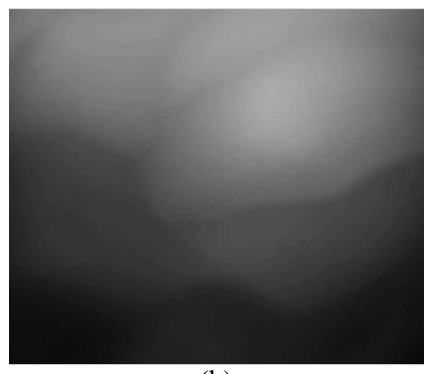

(b)
Figure 5. Shows colour (a) and greyscale (b) images acquired by the system 


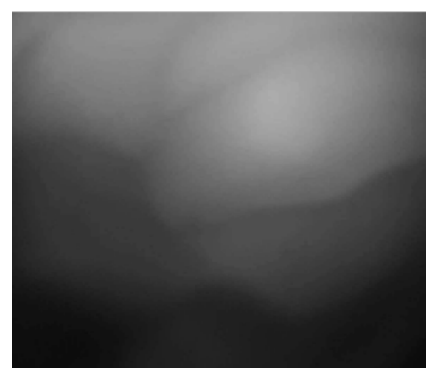

(a)

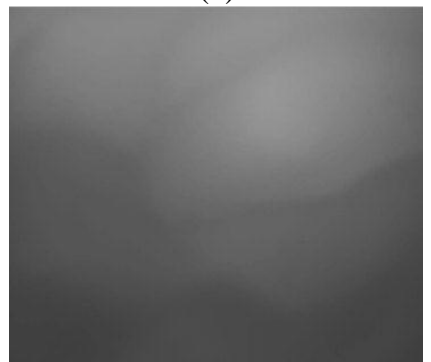

(d)

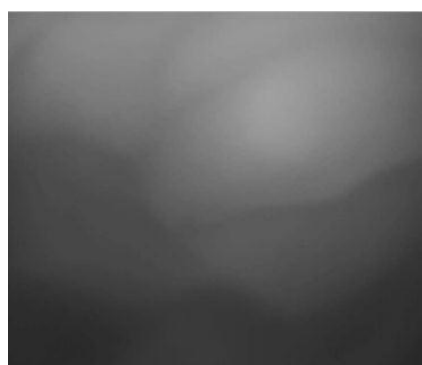

(b)

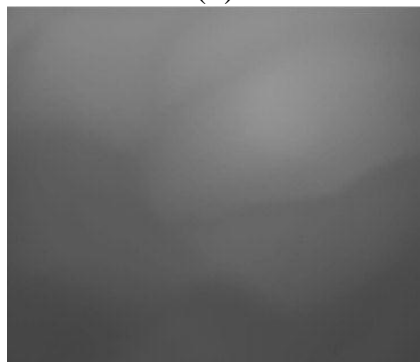

(e)

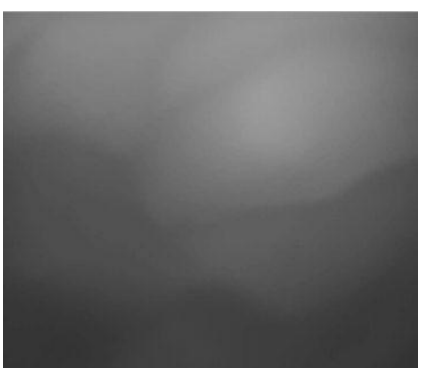

(a)

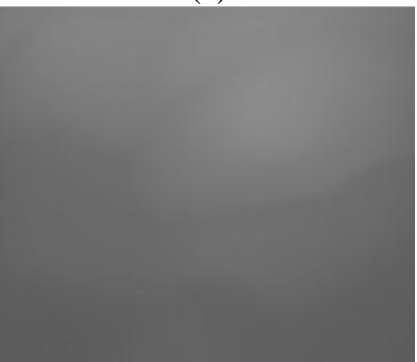

(f)

Figure 6. Grayscale images acquired from different distances: (a) $5 \mathrm{~cm}$, (b) $10 \mathrm{~cm}$, (c) $15 \mathrm{~cm}$, (d) 20 , (e) 25 , (f) $30 \mathrm{~cm}$.

As shown in Figure 6, it can be noted visually that the smaller the distance between the camera and the palm, the better the image quality, especially the quality of the veins (i.e. the emergence of the veins), where a distance of $5 \mathrm{~cm}$ was the optimal distance from which to capture the image. The variance was used to measure the optimal distance between the camera and the palm of the hand, as shown in the following equations [28]:

$\operatorname{Var}=\sigma^{2}$

Eq. 4

where $\sigma$ is the standard deviation, which is defined as follows [25]:

$\sigma=\sqrt{\frac{1}{n} \sum_{i=1}^{n}\left(x_{i}-\bar{x}\right)^{2}}$

Eq. 5

Table 1 represents the statistical properties of the images acquired from different distances, while Figure 7 represents the relationship between variance and distance.

The relationship between variance and distance is inverse, where the greater the distance between the camera and the palm of the hand, the lower the variance. The variance is the average of the squares of the standard deviations of pixels, which represents the average of the mean squared differences from the mean [29]. The greater the distance between the camera and the palm of the hand, the greater the mean values because the values of the minimum value and the maximum value are close, which leads to obscuring the details, especially the veins.
Table 1. The statistical properties of the images acquired from different distances.

\begin{tabular}{cccccc}
\hline \hline Distances (cm) & Min & Max & $\boldsymbol{\mu}$ & $\boldsymbol{\sigma}$ & Var \\
\hline 5 & 10 & 202 & 83.4521 & 46.2023 & 2134.70 \\
10 & 40 & 184 & 94.1006 & 34.1651 & 1167.30 \\
15 & 50 & 178 & 98.1428 & 30.0294 & 901.7677 \\
20 & 61 & 171 & 102.5036 & 25.4563 & 648.0233 \\
25 & 67 & 167 & 104.9600 & 23.1275 & 534.8830 \\
30 & 91 & 152 & 113.5827 & 13.8829 & 192.7338 \\
\hline \hline
\end{tabular}

Where: Min, Max, $\mu, \sigma$, and Var are the minimum, maximum, mean, standard deviation, and variance values, respectively.

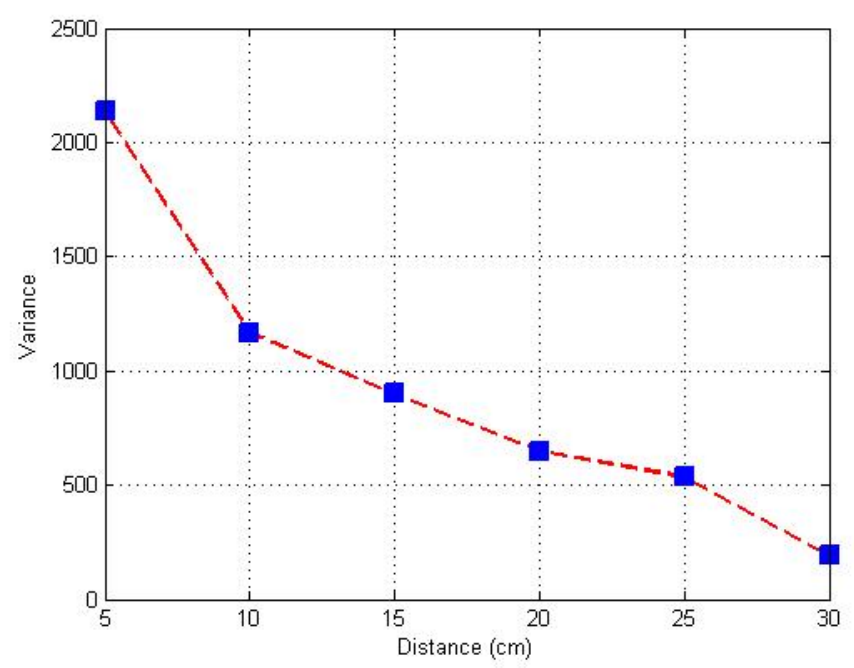

Figure 7. The relationship between variance and distance. 
From the acquired images, we note that superficial veins differ from deep veins in terms of their clarity. The information obtained from veins with large diameters near the surface of the skin helps greatly in understanding the relationship between vein diameter and contrast. There is a linear relationship between the diameter of the vein and its depth [30]. The linear relationship is with respect to veins of large diameters that are found at great depths, but the veins studied in this research take large or relatively small sizes and are in shallow areas in the palm of the hand, that is, they are superficial veins of non-large diameters [31]. It should be noted that the correlation between the contrast and depth or between the contrast and diameter of veins is only a rough guide. The behaviour of variance occurs when the matter is related to one of the two variables (depth or diameter) with the stability of the other variable, where it can be traced by drawing a contrast scheme with one of the transformers by fixing the value of the other variable [32]. Figure 8 depicts a contrast scheme with depth.

The exponential curve shows the decreasing trend, which shows that there is a negative relationship between contrast and vein depth, such that the greater the depth of the vein, the lower the contrast. Figure 9 illustrates the relationship between contrast and vein diameter.

The function of the Wiener filter is to suppress the noise in the images. In other words, when a Wiener filter is applied, its task is to reduce the standard deviation of the processed image of the object. The result of applying the CLAHE technique is shown in Figure 10.

The CLAHE technique results in increased image contrast, especially of the veins. The CLAHE technique works on small areas in the image, called vamps, instead of the whole image. It then combines adjacent vamps, using bilinear interpolation to eliminate industrial boundaries; this can limit contrast, especially in homogeneous areas, to avoid noise in the image [18].

The threshold given in Equation 2 was applied and proved effective in isolating the veins from the rest of the image.
The threshold divides the image into two segments: the segments above the threshold correspond to veins, while segments below the threshold form the rest of the image region. Some undesirable pixels are visible in the result. To remove these areas, we used morphological processes, leading to the image presented in Figure 11.

The threshold divides the image into two segments: the segments above the threshold correspond to veins, while segments below the threshold form the rest of the image region. Some undesirable pixels are visible in the result. To remove these areas, we used morphological processes, leading to the image shown in Figure 12.

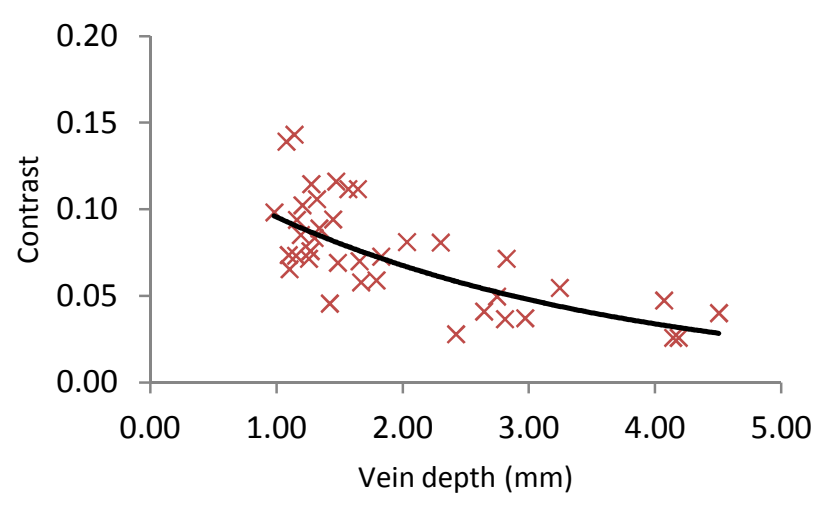

Figure 8. Relationship between contrast and vein depth.

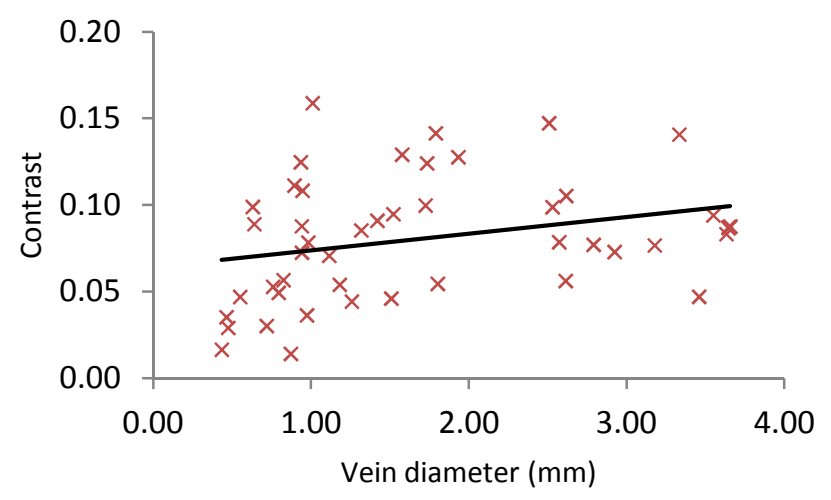

Figure 9. Relationship between contrast and vein diameter.

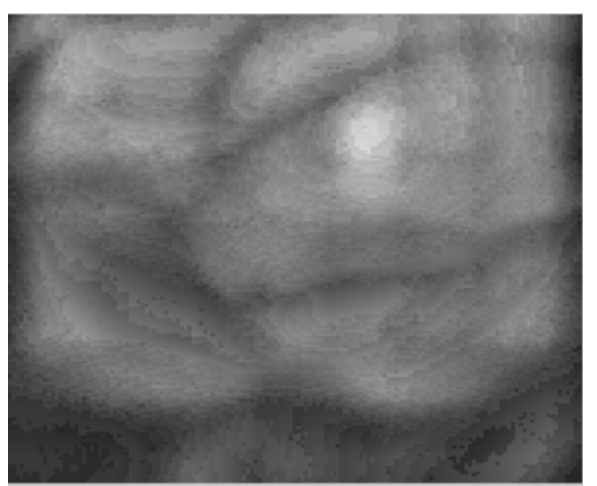

Figure 10. Result after applying the CLAHE technique to the image presented in Figure 5b.

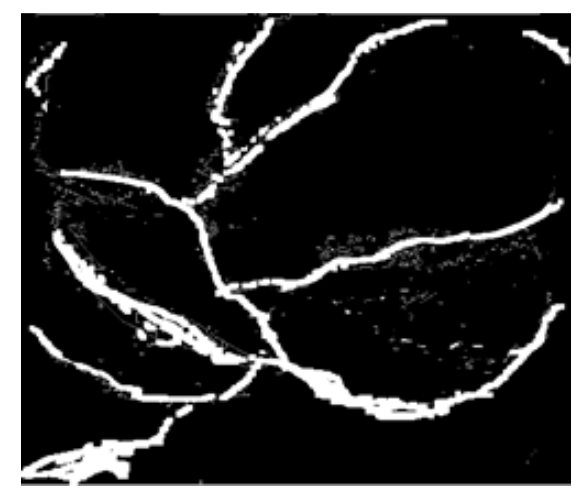

Figure 11. Image after segmentation using the proposed algorithm.

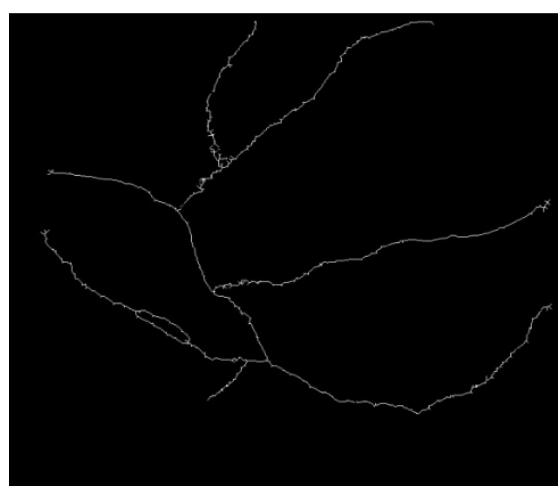

Figure 12. After application of the removing unwanted pixels technique to the image shown in Figure 11. 


\section{Conclusion}

In this paper, a low-cost system designed to detect the veins in the palm of the hand using NIR LEDs and image-processing techniques is presented. The image-processing techniques used here can produce effective and accurate imaging of the veins in the palm. The process of evaluating the functioning of the proposed system involves both physiological aspects of veins and quantitative studies, as this study demonstrates the depth of the vein and contrast needed to clearly detect veins. However, further quantitative and experimental studies are needed to achieve optimal results.

\section{References}

[1] Yen K, Gorelick MH. New biomedical devices that use near-infrared technology to assist with phlebotomy and vascular access. Pediatr Emerg Care. 2013;29(3):383-385.

[2] Francis M, Jose A, Glan Devadhas G, Avinashe KK. A novel technique for forearm blood vein detection and enhancement. Biomedical Research. 2017;28(7):2913-2919.

[3] Scoppettuolo G, Pittiruti M, Pitoni S, et al. Ultrasound-guided "short" midline catheters for difficult venous access in the emergency department: a retrospective analysis. Int J Emerg Med. 2016;9(1):3.

[4] Saito H, Togawa T. Detection of needle puncture to blood vessel using puncture force measurement. Med Biol Eng Comput. 2005;43(2):240-244.

[5] Chandra F, Wahyudianto A, Yasin M. Design of vein finder with multi tuning wavelength using RGB LED. J Phys: Conf Ser. 2017;853(1):012019.

[6] Pailler-Mattei C, Bec S, Zahouani H. In vivo measurements of the elastic mechanical properties of human skin by indentation tests. Med Eng Phys. 2008;30(5):599-606.

[7] Vogelmann TC, Bornman JF, Josserand S. Photosynthetic light gradients and spectral regime within leaves of Medicago sativa. Phil Trans R Soc Lond B Biols Sci. 1989;323(1216):411-421.

[8] Zhang EZ, Povazay B, Laufer J, et al. Multimodal photoacoustic and optical coherence tomography scanner using an all optical detection scheme for 3D morphological skin imaging. Biomed Opt Express. 2011;2(8):2202-2215.

[9] Fu D, Ye T, Matthews TE, et al. High-resolution in vivo imaging of blood vessels without labeling. Opt Lett. 2007;32(18):26412643.

[10] Fuksis R, Greitans M, Nikisins O, Pudzs M. Infrared imaging system for analysis of blood vessel structure. Elektronika ir Elektrotechnika. 2010;97(1):45-48.

[11] Nazish S, Zafar A, Shahid R, et al. Relationship between glycated haemoglobin and carotid atherosclerotic disease among patients with acute ischaemic stroke. Sultan Qaboos University Medical Journal. 2018;18(3):e311-e317.

[12] Swarbrick J. Encyclopedia of pharmaceutical technology. CRC Press; 2013.

[13] Kienle A, Lilge L, Vitkin IA, et al. Why do veins appear blue? A new look at an old question. Appl Opt. 1996;35(7):1151-1160.

[14] Bäumler W, Ulrich H, Hartl A, et al. Optimal parameters for the treatment of leg veins using Nd: YAG lasers at $1064 \mathrm{~nm}$. Br J Dermat. 2006;155(2):364-371.

[15] García AM, Horche PR. Light source optimizing in a biphotonic vein finder device: Experimental and theoretical analysis. Results Phys. 2018;11:975-983.

[16] Cholewka A, Kajewska J, Kawecki M,. How to use thermal imaging in venous insufficiency? J Thermal Anal Calorimetry. 2017;130(3):1317-1326.

[17] Helmich FP, Ivison RJ. FIRI—A far-infrared interferometer. Experimental Astronomy. 2009;23(1):245-276.

[18] Ahmed KI, Habaebi MH, Islam R. A Real Time Vein Detection System. Indones J Electr Eng Comput Sci. 2018;10(1):129-137.

[19] Lammertyn J, Peirs A, De Baerdemaeker J, Nicola1 B. Light penetration properties of NIR radiation in fruit with respect to nondestructive quality assessment. Postharvest Biol Technol. 2000;18(2):121-132.

[20] Chandra F, Wahyudianto A, Yasin M. Design of vein finder with multi tuning wavelength using RGB LED. J Phys: Conf Series. 2017;853(1):012019.

[21] Miura N, Nakazaki K, Fujio M, Takahashi K. Technology and future prospects for finger vein authentication using visible-light cameras. Hitachi Review. 2018;67(5):576-577.

[22] Prijono A, Hangkawidjaja AD, Ahmar AS. Verification Image of The Veins on The Back Palm with Modified Local Line Binary Pattern (MLLBP) and Histogram. J Phys: Conf Series. 2018;954(1):012014.

[23] Cui M, Hu J, Razdan A, Wonka P. Color-to-gray conversion using ISOMAP. The Visual Computer. 2010;26(11):1349-1360.

[24] Wu QQ, Lee JP, Park MH, et al. A study on development of optimal noise filter algorithm for laser vision system in GMA welding. Procedia Engineering. 2014;97:819-827. 
[25] Alwazzan MJ, Ismael MA, Hussain MK. Brain Tumour Isolation in MRI Images Based on Statistical Properties and Morphological Process Techniques. J Phys: Conf Series 2019;1279(1):012018.

[26] Kamola G, Spytkowski M, Paradowski M, Markowska-Kaczmar U. Image-based logical document structure recognition. Pattern Analysis and Applications. 2015;18(3):651-665.

[27] Loomis WF. Skin-Pigment Regulation of Vitamin-D Biosynthesis in Man: Variation in solar ultraviolet at different latitudes may have caused racial differentiation in man. Science. 1967;157(3788):501-506.

[28] Wang Y, Yang J, Yin W, Zhang Y. A new alternating minimization algorithm for total variation image reconstruction. SIAM J Imaging Sci. 2008;1(3):248-272.

[29] Battese GE, Harter RM, Fuller WA. An error-components model for prediction of county crop areas using survey and satellite data. Journal of the American Statistical Association. 1988;83(401):28-36.

[30] Witting MD, Schenkel SM, Lawner BJ, Euerle BD. Effects of vein width and depth on ultrasound-guided peripheral intravenous success rates. J Emerg Med. 2010;39(1):70-75.

[31] Soujanya G. Depth and size limits for the visibility of veins using the VeinViewer imaging system. Master of Science Thesis, The University of Tennessee, Memphis, USA. 2007.

[32] Al-Harosh MB, Shchukin SI. Peripheral vein detection using electrical impedance method. J Electrical Bioimpedance. 2019;8(1):7983. 